\title{
The influence of overlapping response sets on task inhibition
}

\author{
Miriam Gade \\ Max Planck Institute for Human Cognitive and Brain Sciences, Leipzig, Germany \\ AND \\ IRING KOCH \\ RWTH University Aachen, Aachen, Germany
}

\begin{abstract}
The aim of the present study was to examine the contribution of overlapping response sets to the occurrence of task inhibition, measured as $n-2$ task repetition cost. We had subjects switch among four tasks. Three tasks overlapped on both stimulus set and response set. A fourth, neutral, univalent task never overlapped on the stimulus set but, across three experiments, varied in terms of the degree of overlap in the response set. We found that overlap in response set affects task inhibition. We suggest that response set overlap increases the competition among tasks and, thus, triggers task inhibition to resolve this competition.
\end{abstract}

To guarantee successful performance in sequences of tasks, it is commonly assumed that control processes monitor performance and ensure that we attain our intended goals (Botvinick, Braver, Barch, Carter, \& Cohen, 2001). These control processes are postulated to exert their influence by altering the level of activation and inhibition of the cognitive representations of the different tasks. These representations have been termed task set (e.g., Rogers \& Monsell, 1995).

Common definitions of task sets postulate that a task set comprises, on the one hand, stimulus-related processes, such as stimulus encoding and identification. On the other hand, response-related processes, such as relevant stimulus-response ( $\mathrm{S}-\mathrm{R}$ ) mappings and activation of the correct response category (i.e., left or right) or modality (i.e., finger or foot), are also part of a task set (Meiran, 2000; Rogers \& Monsell, 1995; Philipp \& Koch, 2005; Schuch \& Koch, 2004). This multicomponent structure is also reflected in the model of Meiran, who explicitly postulated separate stimulus and response sets. The response set is thought to represent the task-specific S-R mappings. An $\mathrm{S}-\mathrm{R}$ mapping constitutes the cognitive meaning of a response, which is the link between a stimulus and a response. For example, when a red stimulus requires a left keypress in the context of a color judgment, the meaning of a left keypress is red. However, in another task, such as size judgment, the meaning of a left keypress may be small, when subjects are supposed to press a left key when a small stimulus is given. According to the model of Meiran, the stimulus set is likely to be sensitive to (intentional) changes of activation (e.g., biasing), whereas the response set is influenced mainly by the last executed response and cannot be prepared beforehand.
Several authors have suggested that inhibition is necessary to guarantee successful selection and implementation of task sets (e.g., Allport \& Wylie, 1999; Goschke, 2000; Masson, Bub, Woodward, \& Chan, 2003). By using a paradigm with three different tasks, several studies recently showed that inhibition indeed plays a role in the successful selection and implementation of required task sets (see, e.g., Arbuthnott, 2005; Gade \& Koch, 2005; Hübner, Dreisbach, Haider, \& Kluwe, 2003; Koch, Gade, \& Philipp, 2004; Mayr \& Keele, 2000; Philipp \& Koch, 2005; Schuch \& Koch, 2003).

In these studies, task inhibition is inferred from the presence of an $n-2$ repetition cost. The $n-2$ repetition cost is observed when impaired performances are found in $n-2$ task repetitions (ABA), relative to $n-2$ task switches (i.e., $\mathrm{CB} A$ ). The $n-2$ repetition cost is assumed to arise because the no longer relevant task set (i.e., the task set that was used one trial before) is inhibited, and this inhibition persists over one or more intervening trials (e.g., Mayr \& Keele, 2000; Schuch \& Koch, 2003). Thus, when subjects are required to switch back to a recently performed task, they have to overcome this residual inhibition, and this is reflected in higher latencies and error rates, the $n-2$ repetition cost.

It is still an open issue under which conditions task inhibition is observed and which parts of the task set are targets for this inhibition process. Recent evidence suggests that the inhibition effect is modulated by the time elapsing between two tasks (Gade \& Koch, 2005), explicit knowledge about task sequences (Koch, Philipp, \& Gade, 2006; Mayr \& Keele, 2000), the occurrence of task repetitions within a sequence (Philipp \& Koch, 2006), and the type of

I. Koch, koch@psych.rwth-aachen.de 
cue used (Arbuthnott, 2005), as well as the need to select a response on a given trial (Schuch \& Koch, 2003).

The above-mentioned study by Schuch and Koch (2003) also provides some hints as to which parts of a task set might be a potential target of task inhibition. In their study, Schuch and Koch (2003) used a go/no-go paradigm to show that task inhibition was observed primarily when subjects selected a response in trial $n-1$. If trial $n-1$ was a no-go trial, the $n-2$ repetition cost was strongly reduced. In further experiments, Schuch and Koch (2003) concluded that task inhibition arises mainly because of competition among tasks during response selection for the task in trial $n-1$. This competition arises because of persisting activation of the last executed stimulus category response rule. To resolve this competition, the last executed category-response-mapping rule is inhibited (Schuch \& Koch, 2003). There is thus some evidence that task inhibition resolves competition among tasks.

The aim of the present study was to provide further evidence that task inhibition is related to between-task competition due to overlap in response sets, containing all possible S-R mappings, among different tasks. Thus, our goal was to provide direct evidence for the linkage of between-task competition, task inhibition, and overlapping response sets.

To this end, we designed four different tasks. Three of these tasks used multivalent stimuli, in the sense that the stimulus did not indicate which task to perform (e.g., a small, blue letter A; see below). Furthermore these tasks also overlapped in response sets (i.e., they shared the same two vocal responses). These three tasks are referred to as trivalent tasks (T). Importantly, we also used a neutral task that involved univalent stimuli and did not overlap in response set with the other three tasks. This neutral task will henceforth be referred to as the univalent task (U).

Most previous studies that have reported $n-2$ repetition costs have used completely overlapping response sets, as well as completely overlapping stimulus sets (e.g., Gade \& Koch, 2005; Mayr, 2001; Mayr \& Kliegl, 2003; Philipp \& Koch, 2006; Schuch \& Koch, 2003). Thus, it is not clear whether inhibition arises because of overlap in stimulus set or because tasks overlap in their response sets. With our novel design, we tested separately the contribution of overlap in stimulus set and overlap in response set to the occurrence of task inhibition.

To do so, we compared the $n-2$ repetition cost in triplets that consisted of the overlapping tasks only (i.e., TTT) with that in triplets of tasks that contained the univalent task in trial $n-1$ (i.e., TUT; see Table 1). Thus, we com- pared triplets that had the same task in trial $n$, which was always one of the trivalent tasks. However, the triplets differed in the task in trial $n-1$. This could be either another trivalent task for the TTT triplets or the univalent task in the case of the TUT triplets. Given the finding of Schuch and Koch (2003), we expected $n-2$ repetition cost only for the TTT triplets, because there should be competition due to the last performed stimulus category response rule.

Following the account of Schuch and Koch (2003), competition among tasks is likely to arise during the response process. We argue that in the TTT, triplet competition among tasks should arise because of the persisting activation of the last executed task set, including the last used S-R mapping. For successful response selection in trial $n-1$, this last $\mathrm{S}-\mathrm{R}$ mapping has to be inhibited. Therefore, we predicted $n-2$ repetition cost for the comparison of $n-2$ repetitions with $n-2$ switches in the TTT triplets.

When switches to the univalent tasks occur-that is, in the TUT triplets-this $n-2$ repetition cost should not be observed, because of the distinct response set of the univalent task. Since there is no overlap in the response set, task set selection does not require inhibition of the preceding $\mathrm{S}-\mathrm{R}$ mapping. Please note also that all competition due to overlap in stimulus set should be reduced in the TUT triplet. Thus, one might speculate that due to the residual activation of the T task in TUT triplets, as well as the decrease in competition among tasks when switches to the univalent task occur, an $n-2$ repetition benefit could be observable, rather than only no $n-2$ repetition cost.

\section{Overview of Experiments}

We will report three experiments in which the role of response set overlap for task inhibition was further examined. In all the experiments, the subjects performed the four tasks (i.e., the three trivalent tasks, as well as the univalent task) in an unpredictable sequence. All the tasks involved a judgment on the type, size, color, or fill of a given stimulus.

Vocal responses were given for all four tasks. The three trivalent tasks always required a "left" versus "right" vocal response. For the univalent task, we manipulated response set overlap in three steps. In Experiment 1, the subjects gave highly compatible responses by simply naming the stimulus attribute (i.e., "full" vs. "empty"). In Experiment 2, the subjects were required to use arbitrary, also nonoverlapping S-R mappings for the univalent task (i.e., "up" vs. "down"). In the third experiment, we then assessed the influence of full overlap in response set for

Table 1

Order of Tasks in TTT and TUT Triplets for

$n-2$ Task Repetitions and $n-2$ Switches

\begin{tabular}{lll}
\hline & \multicolumn{1}{c}{ TTT } & \multicolumn{1}{c}{ TUT } \\
\hline$n-2$ repetition & $\{$ FSF, FCF, SFS, SCS, CFC, CSC $\}$ & $\{$ FUF, SUS, CUC $\}$ \\
$n-2$ switch & $\{$ CSF, SCF, CFS, FCS, SFC, FSC $\}$ & $\{$ SUF, CUF, FUS, CUS, FUC, SUC \\
\hline
\end{tabular}

Note-F refers to form judgment, $\mathrm{S}$ to size judgment, and $\mathrm{C}$ to color judgment in the trivalent task (T). U refers to the univalent task. UTU versus TTU triplets were analyzed separately and were not compared with TTT and TUT triplets and are, therefore, not included. 
all four tasks. That is, the subjects gave a "left" versus "right" vocal response not only for the trivalent tasks, but also for the univalent task. In that experiment, the only difference between the $\mathrm{U}$ task and the $\mathrm{T}$ tasks was that the stimulus set of the U task did not overlap with that of the $\mathrm{T}$ tasks.

\section{EXPERIMENT 1}

In Experiment 1, we designed a U task that differed in its response set from the $\mathrm{T}$ tasks. We used the three tasks that were performed on trivalent stimuli and overlapped completely in their response set (i.e., all the tasks required the mapping of a stimulus attribute to left/right vocal responses; T tasks). In the fourth task, which required a decision as to whether a rectangle was filled or empty, the subjects had to respond verbally by saying "full" or "empty" (U task). For this U task, there was no overlap in response set with the other T tasks. Our prediction was straightforward: if overlap in either stimulus set or response leads to task inhibition, we should observe no $n-2$ repetition cost in the TUT triplets.

\section{Method}

Subjects. Forty-eight subjects participated (28 of them female; mean age, 24.35 years) and were paid $€ 7$.

Stimuli and Tasks. The stimuli were presented in the center of a 15-in. color monitor connected to an IBM-compatible PC. The stimuli were the letter A and the digit 4, colored either red or blue, with a size of $1.0 \mathrm{~cm}$ (large) or $0.5 \mathrm{~cm}$ (small). The combination of the three different stimulus dimensions (i.e., form, color, and size) resulted in eight different, overlapping stimuli. The stimuli were presented within a white rectangle $(4.0 \mathrm{~cm}$ high and $3.5 \mathrm{~cm}$ wide) that served as cue frame. Four task cues were presented surrounding the rectangle, indicating the three different judgment tasks: four $\$$ signs (1.0 cm high) for form (A vs. 4), four arrows pointing upward and downward $(0.8 \mathrm{~cm}$ high) for size (small vs. large), and four yellow squares $(0.5 \mathrm{~cm}$ high) for color (red vs. blue), respectively. The subjects had to map the relevant stimulus attribute (i.e., large) to a vocal left or right response. Response times (RTs) were measured using a voice key. The experimenter coded the subjects' responses by using the left and right cursor keys.

As a fourth task, the subjects saw a rectangle $(0.5 \mathrm{~cm}$ high and $2.5 \mathrm{~cm}$ wide). For this task, four pound signs (\#) surrounded the cue frame and served as cues. The task was to decide whether the rectangle was filled or empty and to verbalize their decision (i.e., say "full" or "empty"). Again, the experimenter coded the subjects' responses in the same way as that for the T tasks. Testing took place in a dimly lit room. Viewing distance was about $50 \mathrm{~cm}$.

Procedure. The experiment was run in a single session with 1 subject at a time and took about $45 \mathrm{~min}$. Written instructions appeared on the screen, and the experiment was also explained orally. The subjects were informed that they were required to perform four different tasks, depending on the cues surrounding the rectangle in which the stimulus was presented. The S-R mappings were explained for all the tasks. A card containing the specific S-R mapping was placed below the monitor. The subjects performed two practice blocks of 12 trials each to get familiar with the tasks. These practice blocks could be repeated a second time when necessary (i.e., if the subjects had problems in understanding the task requirements). The instructions emphasized both speed and accuracy. The subjects received feedback when they committed an error. The word Fehler (German for error) was displayed for $500 \mathrm{msec}$.

A trial started with a blank screen for $900 \mathrm{msec}$. The rectangle frame appeared together with the cues, followed by the stimulus after $100 \mathrm{msec}$. Stimuli and cues remained on the screen until the response was given. An experimental block of trials consisted of 132 trials, with each task presented equally often. After each block, the subjects received feedback about their mean RT and were invited to take a small break before starting with the next block. Altogether, the subjects performed six experimental blocks, followed by a postexperimental interview. The postexperimental interview probed the subjects about whether they had noted anything special or had had some kind of strategy to deal with the four tasks.

Design. The two independent variables were task triplet (TTT vs. TUT $)^{1}$ and task sequence ( $n-2$ repetition vs. $n-2$ switch). Immediate task repetitions did not occur. The task sequence was pseudorandom, with the constraint that each task and each task triplet occurred equally often within one block. The stimulus and response sequence was random, with the constraint that each stimulus appeared equally often within one block. Direct stimulus repetition did not occur. All eight possible S-R mappings were used, counterbalanced across subjects. The dependent variables were mean RT and error rate. Alpha level was set to .05 .

\section{Results}

In Experiment 1, only correct trials that were preceded by at least two correct trials were included in the mean RT analysis. The RT analysis was conducted with an ANOVA, using task triplet (TTT vs. TUT) and task sequence $(n-2$ repetition vs. $n-2$ switch) as independent variables (see Table 2). All reactions below $200 \mathrm{msec}$ and above two standard deviations from mean RT were regarded as outliers and were not analyzed (3.6\% of otherwise correct trials).

For the RT analysis, the ANOVA yielded a significant interaction between task triplet and task sequence $[F(1,47)=$ $12.32, p<.05$, partial $\left.\eta^{2}=.208\right]$. An $n-2$ repetition cost was observed only for the TTT triplets $[M=39 \mathrm{msec}$; $t(1,47)=3.86, p<.005$; see Table 2], but not for the TUT triplets $[M=-20 \mathrm{msec} ; t(1,47)=-1.5, p=.15]$. There was a significant main effect of task triplet $[F(1,47)=6.11, p<$ .05 , partial $\left.\eta^{2}=.115\right]$. The subjects were slower for TUT triplets than for TTT triplets (1,396 vs. 1,370 msec). The main effect of task sequence was not significant $(F<1.1)$.

In Experiment 1, the overall error rate was $4.6 \%$ (see Table 3). As in the RT data, task triplet interacted significantly with task sequence $[F(1,47)=5.26, p<.05$, par-

Table 2

Mean Response Times (in Milliseconds, With Standard Deviations) as a Function of Task Sequence ( $n-2$ Repetition vs. $n-2$ Switch) and Task Triplet (TTT vs. TUT) for Experiments 1-3

\begin{tabular}{|c|c|c|c|c|c|c|c|c|c|c|c|c|}
\hline & \multicolumn{4}{|c|}{ Experiment 1} & \multicolumn{4}{|c|}{ Experiment 2} & \multicolumn{4}{|c|}{ Experiment 3} \\
\hline & \multicolumn{2}{|c|}{ TTT } & \multicolumn{2}{|c|}{ TUT } & \multicolumn{2}{|c|}{ TTT } & \multicolumn{2}{|c|}{ TUT } & \multicolumn{2}{|c|}{ TTT } & \multicolumn{2}{|c|}{ TUT } \\
\hline & $M$ & $\overline{S D}$ & $M$ & $S D$ & $M$ & $\overline{S D}$ & $M$ & $S D$ & $M$ & $\overline{S D}$ & $M$ & $S D$ \\
\hline$n-2$ repetition & 1,389 & 225 & 1,386 & 205 & 1,440 & 254 & 1,481 & 255 & 1,461 & 247 & 1,497 & 248 \\
\hline$n-2$ switch & 1,350 & 216 & 1,406 & 239 & 1,426 & 236 & 1,501 & 272 & 1,421 & 222 & 1,464 & 234 \\
\hline
\end{tabular}

Note-T, trivalent task; U, univalent task. 
Table 3

Errors (in Percentages, With Standard Deviations) as a Function of Task Sequence (n-2 Repetition vs. $n-2$ Switch) and Task Triplet (TTT vs. TUT) for Experiments 1-3

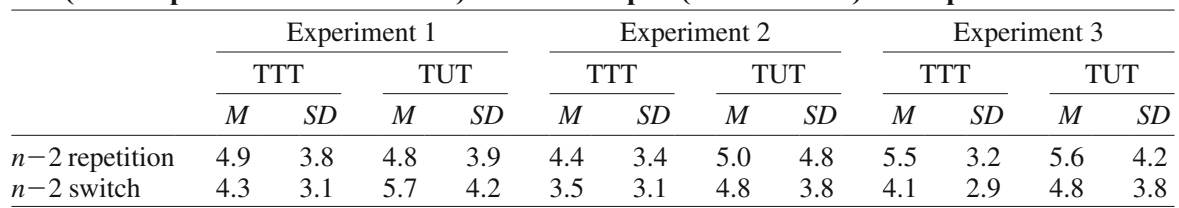

Note- $\mathrm{T}$, trivalent task; $\mathrm{U}$, univalent task.

tial $\left.\eta^{2}=.101\right]$. This interaction was due to a small $n-2$ repetition cost for the TTT triplets (4.9\% vs. $4.3 \%)$ and a slightly reversed $n-2$ repetition benefit for the TUT triplets $(4.8 \%$ vs. $5.7 \%$; both $t \mathrm{~s}<1.6$, both $p \mathrm{~s}>.13)$.

\section{Discussion}

In Experiment 1, we showed that inhibition between tasks is modulated by overlap among tasks. The subjects had no $n-2$ repetition cost when the recently performed task did not overlap in stimulus set or response set. We argue that the overlap in stimulus set, as well as the overlap in response set, increases competition among tasks. Inhibition is then needed to resolve this between-task competition (Gade \& Koch, 2005).

Note that the S-R mapping for the U task was natural (i.e., verbalization of the perceptual feature of the stimulus), whereas the T tasks used arbitrary S-R mappings. To rule out the possibility that arbitrariness of S-R mappings per se plays a role and to further demonstrate that response set overlap triggers task inhibition, we sought to replicate the results of Experiment 1 with an arbitrary $S-R$ mapping for the $\mathrm{U}$ task.

\section{EXPERIMENT 2}

In Experiment 2, we further tested our claim that overlap of elements among response sets triggers task inhibition. Experiment 2 was analogous to Experiment 1, but the difference was that the S-R mapping for the $U$ task was arbitrary and no longer contained stimulus attributes. That is, the subjects were now required to say "up" versus "down" as responses in the U tasks. The responses for the $\mathrm{T}$ tasks remained the same as those in Experiment 1 (i.e., "left" vs. "right" vocal responses).

\section{Method}

Subjects. Forty-eight new subjects ( 36 of them female; mean age, 25.75 years) participated in Experiment 2 and were paid $€ 7$.

Stimuli, Tasks, and Procedure. The stimuli, tasks, and procedure were the same as those in Experiment 1. The only difference was that the subjects were now required to say "up" or "down" (counterbalanced across subjects) as a response in the U task.

Design. The two independent variables were task triplet (TTT vs. TUT) and task sequence ( $n-2$ repetition vs. $n-2$ switch). The dependent variables were mean RT and error rate. Alpha level was set to .05 .

\section{Results}

The RT analysis was conducted with an ANOVA using task triplet (TTT vs. TUT) and task sequence $(n-2$ repeti- tion vs. $n-2$ switch) as independent variables. All reactions below $200 \mathrm{msec}$ and above two standard deviations from mean RT were regarded as outliers and were not analyzed (3.2\% of otherwise correct trials).

For the RT analysis, the predicted interaction between task triplet and task sequence was significant $[F(1,47)=$ $4.20, p<.05$, partial $\left.\eta^{2}=.082\right]$. Contrary to the results of Experiment 1 , the $n-2$ repetition cost for the TTT triplets failed to reach significance $[M=14 \mathrm{msec} ; t(1,47)=1.44$, $p=.16]$, but we observed a trend toward the expected direction. For the TUT triplets, a nonsignificant $n-2$ repetition benefit occurred $[M=-20 \mathrm{msec} ; t(1,47)=1.41$, $p=.17$; see Table 2].

The ANOVA also yielded a significant main effect for task triplet $\left[F(1,47)=35.62, p<.05\right.$, partial $\left.\eta^{2}=.431\right]$. Again, the subjects were faster for TTT triplets than for TUT triplets $(1,434$ vs. $1,491 \mathrm{msec})$. The main effect of task sequence was not significant $(F<1)$.

The overall error rate in Experiment 2 was $3.8 \%$ (see Table 3). The interaction between task triplet and task sequence was not significant in the error data $(F<1)$. As in the RT data, the main effect of task triplet was significant $\left[F(1,47)=8.8, p<.05\right.$, partial $\left.\eta^{2}=.158\right]$. The subjects made fewer errors in trivalent tasks when these were preceded by another trivalent task, as compared with when these were preceded by the univalent task (4.0\% vs. $4.9 \%)$. Contrary to the RT data, task sequence showed a marginally significant main effect $[F(1,47)=3.03, p=$ .088 , partial $\left.\eta^{2}=.061\right]$. Corroborating the nonsignificant $n-2$ repetition cost for the TTT triplets in the RT data, we observed a significant $n-2$ repetition cost of $0.9 \%$ in the error data $[t(1,47)=2.32, p=.025]$. There was also a small $n-2$ repetition cost for the TUT triplets $(0.2 \%)$ that was not significant $(t<1)$.

\section{Discussion}

Experiment 2 confirmed the results of Experiment 1 in that nonoverlapping response sets abolished the $n-2$ repetition cost also with arbitrary S-R mappings. Note that we found an $n-2$ repetition cost for the TTT triplets in both the RT and the error data (even though it was not significant in the RT data). For the TUT triplet, there was a trend toward an $n-2$ repetition benefit in the RT data, but not in the error data. Thus, we conclude that competition among tasks due to overlap between response sets is abolished even when arbitrary S-R mappings are used in all the tasks.

Schuch and Koch (2003) argued that competition among tasks arises mainly during response processes. According to their view, the "cognitive meaning" of the last re- 
sponse (i.e., left-red and right-blue) persists and hampers the selection of the currently relevant response set (i.e., left-small and right-big). They assumed that inhibition resolves this competition and is exerted to the no longer relevant response set. However, Schuch and Koch (2003) used tasks that overlapped not only in response sets, but also in stimulus sets (i.e., by using multivalent stimuli). Thus, it remains to be determined whether inhibition occurs only because of interfering response sets or whether overlapping stimulus sets also contribute to the occurrence of inhibition. Ruling out overlap in the stimulus set as alternative explanation is also necessary to validate the conclusions drawn from Experiments 1 and 2 so far.

\section{EXPERIMENT 3}

Experiment 3 was designed to control for overlap in stimulus set, which might also lead to task inhibition. Thus, in Experiment 3, we used the same paradigm as that in Experiments 1 and 2, but this time the response sets overlapped in all four tasks. To this end, the filled-empty judgment was mapped onto vocal left/right responses as in the other three tasks. Our predictions were clear-cut. If overlap in response sets alone contributes to the occurrence of task inhibition, we should find an $n-2$ repetition cost for both the TTT and TUT task triplets. If, however, overlap in stimulus set is critical, we should still see no $n-2$ repetition cost for TUT triplets.

\section{Method}

Subjects. Forty-eight new subjects participated (35 of them female; mean age, 24.6 years) and were paid $€ 7$.

Stimuli, Task, Procedure, and Design. The stimuli, tasks, and procedure were the same as those in Experiments 1 and 2. The subjects always used a vocal "left" or "right" response also for the univalent task. The independent variables were task triplet (TTT vs. TUT) and task sequence ( $n-2$ repetition vs. $n-2$ switch).

\section{Results}

RTs and errors were analyzed as in Experiments 1 and 2 . We discarded $1.1 \%$ of otherwise correct trials as outliers.

For the RT analysis, the interaction between task triplet and task sequences was far from significance $(F<1)$. We found a significant main effect of task triplet $[F(1,47)=$ $15.51, p<.05$, partial $\left.\eta^{2}=.248\right]$. Again, the subjects were faster for TTT triplets than for TUT triplets (1,441 vs. $1,481 \mathrm{msec})$. Task sequence also yielded a significant main effect $\left[F(1,47)=11.99, p<.05\right.$, partial $\left.\eta^{2}=.203\right]$. The subjects exhibited an overall $n-2$ repetition cost of $36 \mathrm{msec}$. Further post hoc test showed a significant $n-2$ repetition cost for the TTT triplet $[M=40 \mathrm{msec}$; $t(1,47)=3.38, p<.001]$. For the TUT triplet, we also found a significant $n-2$ repetition cost $[M=33 \mathrm{msec}$; $t(1,47)=2.31, p=.025 ;$ see Table 2].

The overall error rate in Experiment 3 was $4.5 \%$ (see Table 3). As in the RT data, there was no significant interaction between task triplet and task sequence $(F<1)$. We found no significant main effect of task triplet $(F<1)$. We obtained a significant main effect only for task sequence $\left[F(1,47)=5.78, p<.05\right.$, partial $\left.\eta^{2}=.109\right]$. The subjects showed an overall $n-2$ repetition cost of $1.1 \%$. Post hoc tests showed a significant $n-2$ repetition cost for the TTT triplets $[M=1.4 \% ; t(1,47)=2.71, p=.009]$, but not for the TUT triplet $[M=0.8 \% ; t(1,47)=1.32, p=.19]$.

\section{Discussion}

Experiment 3 provides clear evidence that overlap in response set leads to the occurrence of task inhibition, reflected in $n-2$ repetition cost. We found an $n-2$ repetition cost for the TTT triplet, as well as for the TUT triplet. Thus, the data of all three experiments indicate that competition among tasks because of overlapping response sets leads to task inhibition. However, up to now, our theoretical conclusions were based on the finding of no $n-2$ repetition cost in TUT triplets in Experiments 1 and 2, relative to Experiment 3. To provide stronger statistical evidence for our conclusion, we performed a between-experiment analysis, comparing both Experiments 1 and 2 (having no overlap in response set) with Experiment 3 (with an overlap in response set). If inhibition is modulated by the competition among tasks, we should expect a three-way interaction between task triplet, task sequence, and experiment.

\section{BETWEEN-EXPERIMENT COMPARISON}

We compared the results of all three experiments with each other in an ANOVA with task triplet (TTT vs. TUT) and task sequence ( $n-2$ repetition vs. $n-2$ switch) as within-subjects variables and overlap as a between-subjects variable (no overlap vs. overlap). For the between-subjects analysis, Experiments 1 and 2 were grouped together.

We found an $n-2$ repetition cost of $40 \mathrm{msec}$ for the TTT triplets and of $33 \mathrm{msec}$ for the TUT triplets in the overlap group. However, there was only an $n-2$ repetition cost of $26 \mathrm{msec}$ for the TTT triplets in the nonoverlap group and no such cost for the TUT triplets $(M=-20 \mathrm{msec})$. The predicted three-way interaction between task triplet, task sequence, and overlap was nearly significant $[F(1,143)=$ $3.879, p=.051$, partial $\left.\eta^{2}=.027\right]$. A closer inspection of the data showed that the three-way interaction was significant for the comparison of Experiments 1 and 3 $\left[F(1,95)=5.11, p<.05\right.$, partial $\left.\eta^{2}=.052\right]$. The comparison of Experiments 2 and 3 did not yield a significant three-way interaction $[F(1,95)=1.37, p=.24]$. This failure to statistically confirm the numerically present difference in the data patterns in Experiments 2 and 3 may be due to the high overall variance in Experiment 2.

Besides a trend toward the three-way interaction, we found a significant interaction between task sequence and overlap $\left[F(1,143)=8.54, p<.05\right.$, partial $\left.\eta^{2}=.047\right]$. That is, we observed an overall $n-2$ repetition cost of $37 \mathrm{msec}$ in the overlap group, whereas the $n-2$ repetition cost was nearly absent $(M=6 \mathrm{msec})$ in the nonoverlap group. A more detailed analysis confirmed this interaction of task sequence and overlap in the single comparison of Experiments 1 and $3[F(1,95)=4.07, p<.05$, partial $\left.\eta^{2}=.041\right]$, as well as in the comparison of Experiments 2 and $3\left[F(1,95)=8.33, p<.05\right.$, partial $\left.\eta^{2}=.081\right]$.

An analysis of error data yielded only a marginally significant interaction between task sequence and overlap 
$[F(1,143)=2.78, p=.098]$. Thus, we can conclude, albeit somewhat cautiously, that response set overlap indeed affects task inhibition.

\section{ANALYSIS OF UTU TRIPLETS}

In this final section, we will report the analysis of the data of the UTU triplets. We assumed that our U task does not lead to any competition with the other three tasks. This assumption leads to the prediction that for triplets that contain an $n-2$ repetition of the $\mathrm{U}$ task (i.e., UTU), there should be no need for inhibition (i.e., no $n-2$ repetition cost for the UTU triplets, as compared with TTU triplets) in Experiments 1 and 2. However, for Experiment 3, we would expect to find $n-2$ repetition cost if overlap in response set alone is sufficient for task inhibition to occur.

We did not observe a significant $n-2$ repetition cost for the univalent task in Experiment 1 in the RT data $[t(1,47)<1 ; 802$ vs. $804 \mathrm{msec}$ for $n-2$ repetition vs. $n-2$ switch; see Table 2]. However, there was indeed a significant $n-2$ repetition benefit for the UTU triplets in the error data $[t(1,47)=2.2, p=.033 ; 2.4 \%$ vs. $3.4 \%]$. A significant $n-2$ repetition benefit for the UTU triplets was also found in Experiment $2[M=-22 \mathrm{msec}, t(1,47)=$ $2.6, p=.012$, for the RT data; and $M=0.02 \%, t(1,47)=$ 1.24 , for the error data].

Contrary to our assumption, we found an $n-2$ repetition benefit in the RT data also in Experiment $3[M=$ $-30 \mathrm{msec} ; t(1,47)=2.71, p=.009]$, but this was accompanied by a significant $n-2$ repetition cost $[M=1.3 \%$; $t(1,47)=2.95, p=.005]$ in the error data. Thus, our assumptions about the UTU triplets were only partially confirmed (i.e., in Experiments 1 and 2), but the interpretation of the results of Experiment 3 is unfortunately marred by a speed-accuracy trade-off.

\section{GENERAL DISCUSSION}

The aim of our study was to examine the relationship of competition among tasks and the occurrence of task inhibition because of overlap in response sets. We developed a new paradigm with four tasks. Three of the tasks overlapped in stimulus set, as well as in response sets (the $\mathrm{T}$ tasks). Our fourth task had univalent stimuli but showed a varying degree of overlap in the response set (U task). In the analysis, we compared task triplets with different degrees of between-task competition (see Table 1). In TTT triplets, we found $n-2$ repetition cost in all three experiments. In TUT triplets (i.e., triplets that contained the univalent task in trial $n-1$ ), we found no $n-2$ repetition cost when this task had no overlapping response sets (Experiments 1 and 2). We did find $n-2$ repetition cost also for these triplets when the response sets for all four tasks overlapped (Experiment 3 ). We argue that the overlap in response set leads to competition among the four tasks and, therefore, inhibition is exerted to resolve this competition during response selection. However, these results are not fully conclusive at this point in time, since we failed to observe the three-way interaction between experiments. Thus, further experiments seem to be desirable.
Overall, our findings are in line with the model of Schuch and Koch (2003), in which it is assumed that persisting activation of the last response set leads to competition among tasks and impairs performance. Due to this competition, inhibition is necessary and is targeted at the last response set. However, our results are not fully conclusive regarding the question of what exactly is inhibited. Although the previously activated response set seems to be a likely candidate for inhibition, it is still unclear whether inhibition applies only to the last activated specific response meaning or to the whole response set of a task. Clearly, further experiments are required to disentangle these possibilities.

There are at least two studies that do not fit with the notion of the abolishment of $n-2$ repetition cost because of nonoverlapping response sets. These are the studies of Arbuthnott and colleagues (Arbuthnott \& Frank, 2000; Arbuthnott \& Woodward, 2002). In their studies, subjects were required to give vocal responses - namely, the attribute of the stimulus category for letters (response: "vowel" vs. "consonant"), digits (response: "odd" vs. "even"), and symbols (response: "maths" vs. "text"). Thus, response sets were different for all three tasks. Although neither study used overlapping stimuli or overlapping response sets, they observed a significant $n-2$ repetition cost. These studies differ from our study in that the subjects always saw all three kinds of stimuli on the screen (i.e., there was a letter, a digit, and a symbol in each trial). Although two of these stimuli were irrelevant to the task at hand, there might have been some priming from these irrelevant stimuli that also activated the given response of the formerly relevant task. This may have led to competition and interference between the three, otherwise nonoverlapping response sets. In our study, the subjects always saw only one stimulus at a time, so that at least for the univalent task, there was no priming for the competing responses. Thus, it may be that although the overlap of response sets is one major source of competition among tasks, bottomup processes, such as the stimulus-based priming of task sets, also modulate the amount of competition between tasks. Stimuli from currently irrelevant tasks may alter the activation of other, not necessarily overlapping response sets by priming the associated tasks. This may increase overall task competition and, therefore, be another condition under which task inhibition is observed.

The stimulus-based priming processes of task sets such as the ones just mentioned could possibly also explain our failure to find an $n-2$ repetition cost for the UTU triplets. In all the experiments, we found inhibition when the subjects switched among the $\mathrm{T}$ tasks, all using overlapping stimuli.

However, in Experiment 3, we failed to find inhibition for tasks with univalent stimuli that did not prime any of the other tasks (even though it completely overlapped in response set; see the analysis of the UTU triplets). Thus, it seems necessary not only to examine the contribution of overlapping response sets for the occurrence of between-task competition and task inhibition, but also to further study the complex priming effects that might also determine the overall amount of between-task competi- 
tion (Koch \& Allport, 2006; Waszak, Hommel, \& Allport, 2003) and contribute to the occurrence of task inhibition.

To summarize our results, using a novel experimental paradigm, we showed that overlap in response sets contributes to the occurrence of between-task competition and task inhibition to resolve this competition. We conclude that overlap in response set influences the occurrence of task inhibition, reflected in an $n-2$ repetition cost.

\section{AUTHOR NOTE}

We thank Ulrich Mayr, Eric Ruthruff, and an anonymous reviewer for helpful comments on a previous version of this article. We are also grateful to Andrea Philipp for helpful discussion. Correspondence concerning this article should be addressed to I. Koch, RWTH Aachen, Department of Psychology, Jaegerstr. 17-19, D-52066, Aachen, Germany (e-mail koch@psych.rwth-aachen.de).

\section{REFERENCES}

Allport, A., \& Wylie, G. (1999). Task-switching: Positive and negative priming of task-set. In G. W. Humphreys, J. Duncan, \& A. Treisman (Eds.), Attention, space, and action: Studies in cognitive neuroscience (pp. 273-296). Oxford: Oxford University Press.

Arbuthnott, K. D. (2005). The influence of cue type on backward inhibition. Journal of Experimental Psychology: Learning, Memory, \& Cognition, 31, 1030-1042.

Arbuthnott, K. [D.], \& Frank, J. (2000). Executive control in set switching: Residual switch cost and task-set inhibition. Canadian Journal of Experimental Psychology, 54, 33-41.

ArbuthnotT, K. D., \& WoodWARD, T. S. (2002). The influence of cuetask association and location on switch cost and alternating-switch cost. Canadian Journal of Experimental Psychology, 56, 18-29.

Botvinick, M. M., Braver, T. S., Barch, D. M., Carter, C. S., \& Cohen, J. D. (2001). Conflict monitoring and cognitive control. Psychological Review, 108, 624-652.

GADE, M., \& KoCH, I. (2005). Linking inhibition to activation in the control of task sequences. Psychonomic Bulletin \& Review, 12, 530-534

GoschKe, T. (2000). Intentional reconfiguration and involuntary persistence in task-set switching. In S. Monsell \& J. Driver (Eds.), Attention and performance XVIII: Control of cognitive processes (pp. 331-356). Cambridge, MA: MIT Press.

Hübner, M., Dreisbach, G., Haider, H., \& Kluwe, R. H. (2003). Backward inhibition as a means of sequential task set control: Evidence for reduction of task competition. Journal of Experimental Psychology: Learning, Memory, \& Cognition, 29, 289-297.

Koch, I., \& AlLPORT, A. (2006). Cue-based preparation and stimulus-based priming of tasks in task switching. Memory \& Cognition, 34, 433-444.

Koch, I., Gade, M., \& PhilipP, A. M. (2004). Inhibition of response mode in task switching. Experimental Psychology, 51, 52-58.
Косн, I., PhilipP, A. M., \& GAdE, M. (2006). Chunking in task sequences modulates task inhibition. Psychological Science, 17, 346-350.

Masson, M. E. J., Bub, D. N., Woodward, T. S., \& Chan, J. C. K. (2003). Modulation of word-reading processes in task switching. Journal of Experimental Psychology: General, 132, 400-418.

MAYR, U. (2001). Age differences in the selection of mental sets: The role of inhibition, stimulus ambiguity, and response-set overlap. Psychology \& Aging, 16, 96-109.

Mayr, U., \& Keele, S. W. (2000). Changing internal constraints on action: The role of backward inhibition. Journal of Experimental Psychology: General, 129, 4-26.

MAYR, U., \& KLIEGL, R. (2003). Differential effects of cue changes and task changes on task-set selection costs. Journal of Experimental Psychology: Learning, Memory, \& Cognition, 29, 362-372.

Meiran, N. (2000). Reconfiguration of stimulus task sets and response task sets during task switching. In S. Monsell \& J. Driver (Eds.), Attention and performance XVIII: Control of cognitive processes (pp. 377-399). Cambridge, MA: MIT Press.

PhILIPP, A. M., \& Koch, I. (2005). Switching of response modalities. Quarterly Journal of Experimental Psychology, 58A, 1325-1338.

PhILIPP, A. M., \& КосH, I. (2006). Task inhibition and task repetition in task switching. European Journal of Cognitive Psychology, 18, 624-639.

Rogers, R. D., \& Monsell, S. (1995). Costs of a predictable switch between simple cognitive tasks. Journal of Experimental Psychology: General, 124, 207-231.

SchUch, S., \& Косн, I. (2003). The role of response selection for inhibition of task sets in task shifting. Journal of Experimental Psychology: Human Perception \& Performance, 29, 92-105.

SCHUCH, S., \& KосH, I. (2004). The costs of changing the representation of action: Response repetition and response-response compatibility in dual tasks. Journal of Experimental Psychology: Human Perception \& Performance, 30, 566-582.

Waszak, F., Hommel, B., \& Allport, A. (2003). Task-switching and long-term priming: Role of episodic stimulus-task bindings in taskshift costs. Cognitive Psychology, 46, 361-413.

\section{NOTE}

1. Please note that the main analysis will not include the comparison of UTU and TTU triplets. Since these triplets are special in their structure, confounding $n-2$ repetitions with the type of task (i.e., UTU and TTU triplets differed in the type of task [T vs. U] on trials $n-2$ and $n$ and not as the TTT and TUT triplets in the type of task on trial $n-1$ ), we will report the results obtained within these triplets separately at the end of the empirical section.

(Manuscript received April 25, 2005; revision accepted for publication April 12, 2006.) 